\title{
Particle Redistribution During Dendritic Solidification of Particle Suspensions
}

\author{
Noah O. Shanti, ${ }^{\star}$ Kiyoshi Araki, ${ }^{\star \star \ddagger}$ and John W. Halloran ${ }^{\dagger, *, \star *}$ \\ Department of Materials Science and Engineering, University of Michigan, Ann Arbor, Michigan 48109
}

\begin{abstract}
Solidification of the liquid medium in ceramic suspensions containing less than a critical volume fraction powder leads to the formation of particle-free dendrites of the frozen medium. These particle-free dendrites create, after sublimation of the frozen vehicle, large dendrite pores. We define the conditions under which particle-free dendrites form, and relate the size and volume fraction of the dendrites to the volume fraction powder and the solidification rate.
\end{abstract}

\section{Introduction}

USPENSION freeze casting is an uncommon but quite attractive

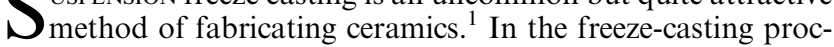
ess, a ceramic powder is suspended in a liquid vehicle. The slurry is poured into a mold, and quickly frozen. The frozen vehicle is then sublimed away (freeze dried), leaving behind a green ceramic body. The green body is then sintered to complete the process. ${ }^{2,3}$ Often, when the slurry is frozen, especially for slurries with a low solid content, the vehicle forms dendrites or other particle-free pockets. During sublimation, these areas become pores in the ceramic. ${ }^{4-6}$ Dendrite pores can be useful as performs for composites 7 or as scaffolds. ${ }^{8}$

During the solidification of the suspension, the freezing vehicle will interact with the suspended particles. Three things may occur: the particles are engulfed by the advancing solid front, the particles are pushed by the advancing front, or the particles are trapped between two solid fronts (i.e., two dendrite arms). This phenomenon has been the focus of much study in metallography, where particles are routinely suspended in molten metals to form dispersion-strengthened materials.

If the particles are engulfed by the solidification front as it advances, a uniform particle distribution will result. If they are rejected, however, the particle concentration in the remaining melt areas will become larger. Araki ${ }^{9}$ has observed that dilute ceramic-camphene suspensions undergo particle segregation during solidification. Dendrites of particle-free camphene grow into the liquid, sweeping the ceramic particles into the interdendritic spaces. Clearly, the particles are being dragged by the moving camphene solid/liquid interface. Eventually, the particle redistribution ceases, and the solid/liquid interface moves into the interparticle spaces, which can be called a "breakthrough" as the solid/liquid interface breaks into the suspension. We want to understand how this occurs, and at what volume fraction particles the breakthrough occurs.

The interaction between particles and an advancing solidification front has been addressed by Uhlmann et al. ${ }^{8}$ considering the pushing of particles by a planar solidification front. We pro-

G. Franks - contributing editor

\footnotetext{
Manuscript No. 21238. Received December 9, 2005; approved March 13, 2006. ${ }^{*}$ Member, American Ceramic Society.

${ }^{\dagger}$ Present address: NGK, Japan.

PAuthor to whom correspondence should be addressed. e-mail: peterjon@umich.edu

${ }^{\star *}$ Fellow, American Ceramic Society.
}

pose that particles are concentrated by this pushing until a "breakthrough" occurs when particles are concentrated enough to resist further concentration. At this point, particles are no longer pushed by the solid front, and the solid/liquid interface breaks past the jammed particles and into the inter-particle spaces. We ask how the volume fraction at "breakthrough," $\Phi_{\mathrm{b}}$, may be related to parameters such as the liquid/solid interfacial energy, total solids content, and particle size.

Particles will be pushed along with the vehicle solid/liquid interface until the capillary drag force pushing the particles with the solid (S)/liquid (L) interface is countered by the force created by the particle concentration. The countering force is the osmotic force from the osmotic pressure of the suspension, $\Pi$. The osmotic pressure of the suspension depends on the volume fraction particles in the suspension $\Phi$, which can be modeled for well-dispersed systems with a modified $^{10}$ Carnahan-Starling equation $^{11}$

$$
\Pi(\phi)=n_{\mathrm{p}} k T\left(\frac{\left(1+\Phi+\Phi^{2}-\Phi^{3}\right)}{\left(\Phi_{\mathrm{m}}-\Phi\right)^{3}}\right)
$$

where $\Phi_{\mathrm{m}}$ is the volume fraction particles at maximum packing, $k$ is Boltzmann's constant, and $n_{\mathrm{p}}$ is the number of particles per unit volume in the suspension. The number of particles can be expressed in terms of the particle volume fraction $\Phi$ and the volume of a single particle $V_{\mathrm{p}}$, so the equation can be expressed as

$$
\Pi(\phi)=\frac{k T}{V_{\mathrm{p}}}\left(\frac{\Phi\left(1+\Phi+\Phi^{2}-\Phi^{3}\right)}{\left(\Phi_{\mathrm{m}}-\Phi\right)^{3}}\right)
$$

Notice that the behavior of this function is to increase slowly with $\Phi$ as the suspension becomes more concentrated, and then rise very rapidly as $\Phi$ approaches the value of $\Phi_{\mathrm{m}}$ when the denominator vanishes and $\Pi\left(\Phi_{\mathrm{m}}\right)$ becomes infinite at $\Phi=\Phi_{\mathrm{m}}$. The osmotic pressure diverges when the particle packing is as high as it can possibly be, such that further concentration is not possible.

For the $\mathrm{S} / \mathrm{L}$ interface to break through the spaces between the collected particles, the pressure must exceed the capillary pressure $\left(P_{\text {cap }}\right)$ required to force an interface into a packing of particles. This is analogous to the capillary pressure at the water/air interface for the problem of drying, which was treated by Smith et $a .^{12}$

$$
P_{\text {cap }}=\frac{3 \Phi \gamma}{(1-\Phi) R}
$$

where $\gamma$ is the liquid-solid interfacial energy for the suspension medium and $R$ is the surface-area equivalent spherical radius for the particles.

Particle drag will concentrate the suspension in the remaining liquid until the volume of fraction particles reaches a critical value $\Phi_{\mathrm{b}}$, where the osmotic pressure exceeds the capillary pressure, so that breakthrough occurs when

$$
\left[\frac{k T}{V_{\mathrm{p}}}\right]\left(\frac{\Phi_{\mathrm{b}}\left(1+\Phi_{\mathrm{b}}+\Phi_{\mathrm{b}}^{2}-\Phi_{\mathrm{b}}^{3}\right)}{\left(\Phi_{\mathrm{m}}-\Phi_{\mathrm{b}}\right)^{3}}\right)=\frac{3 \Phi_{\mathrm{b}} \gamma}{\left(1-\Phi_{\mathrm{b}}\right) R}
$$


This can be re-arranged into

$$
\begin{aligned}
& \frac{\left(\Phi_{\mathrm{m}}-\Phi_{\mathrm{b}}\right)^{3}}{\left(1-2 \Phi_{\mathrm{b}}^{3}+\Phi_{\mathrm{b}}^{4}\right)}=\frac{k T R}{3 V_{\mathrm{p} \gamma}}=W^{3} \\
& \Phi_{\mathrm{m}}-\Phi_{\mathrm{b}}=W\left(1-2 \Phi_{\mathrm{b}}^{3}+\Phi_{\mathrm{b}}^{4}\right)^{1 / 3}
\end{aligned}
$$

For values of $\Phi_{\mathrm{b}}$ around 0.5 , which is relevant in this case, the term $\left(1-2 \Phi_{\mathrm{b}}^{3}+\Phi_{\mathrm{b}}^{4}\right)^{1 / 3} \cong 1$, so the critical particle volume fraction $\Phi_{\mathrm{b}}$ where particle pushing stops and the $\mathrm{S} / \mathrm{L}$ interface moves into the particle-filled region is

$$
\Phi_{\mathrm{b}} \approx \Phi_{\mathrm{m}}-W
$$

with

$$
W=\left(\frac{k T R}{3 V_{\mathrm{p}} \gamma}\right)^{1 / 3}
$$

or for uniform spherical particles

$$
W=\left(\frac{k T}{4 \pi R^{2} \gamma}\right)^{1 / 3}
$$

We expect for suspensions that the critical particle volume fraction where drag ceases will be near close packing: $\Phi_{\mathrm{b}} \cong \Phi_{\mathrm{m}}$. Breakthrough occurs when the particles approach the maximum packing, or simply stated, breakthrough occurs at jamming, with relatively little influence of particle size and surface tension expressed by Eq. (9).

We also predict that breakthrough is independent of the average solid content $\Phi$, so that the volume fraction of dendritic pores should be proportional to $\Phi_{\mathrm{m}}$. So we expect no large dendritic pores if the solids loading of the suspension is greater than or equal to $\Phi_{\mathrm{m}}$. A plot of volume fraction of large dendritic pores after sintering versus the particle solid loading in the suspension before freezing should be linear for low concentrations. It should extrapolate to zero large dendritic porosity when $\Phi=\Phi_{\mathrm{m}}$. The volume fraction $\Phi_{\mathrm{m}}$ should be approximately the same as observed for the viscous flow for suspensions fit to the Krieger-Dougherty equation.

The sizes of the dendritic pores are also of interest. Dendrite size is commonly characterized by the secondary dendrite arm spacing (SDAS), $\lambda_{2}$, which depends on the solidification velocity $v$ by a power law ${ }^{13}$

$$
\lambda_{2}=B v^{-n}
$$

where $B$ and $n$ are constants, with the typical ${ }^{14}$ value for the exponent $n$ being 0.33 .

\section{Experimental Procedure}

We conducted freeze casting of alumina $\left(\mathrm{Al}_{2} \mathrm{O}_{3}\right)$ using camphene as a vehicle. Camphene makes for a very suitable vehicle because it is solid at room temperature $\left(\mathrm{T}_{\mathrm{m}} \sim 44^{\circ}-48^{\circ} \mathrm{C}\right)$, has low liquid viscosity, a high solid vapor pressure, little shrinkage upon solidification, is nontoxic, and is inexpensive. Camphene sublimes quickly and easily at room temperature and ambient pressure. Its solidification behavior is well understood. ${ }^{15}$ The processing details are presented elsewhere. 9 Briefly, the alumina powder was an $\alpha-\mathrm{Al}_{2} \mathrm{O}_{3}$ powder (AG16, Alcoa Chemical, Pittsburgh, PA) with a median size $\left(d_{50}\right)$ of $400 \mathrm{~nm}$ and a specific surface area of $8.6 \mathrm{~m}^{2} / \mathrm{g}$ (from the manufacturer's specifications). The vehicle was $( \pm)$-camphene $\left(\mathrm{C}_{10} \mathrm{H}_{16}, \mathrm{CAS} 79-92-5\right.$, Alfa Aesar/ Avocado Organics, Ward Hill, MA). The dispersant for alumina in liquid camphene at $55^{\circ} \mathrm{C}$ was an amine derivative of a fatty acid condensation polymer (Perfad 9100, UniQema, Everburg, Belgium). After solidification, the frozen camphene was removed by freeze drying at room temperature in ambient air.

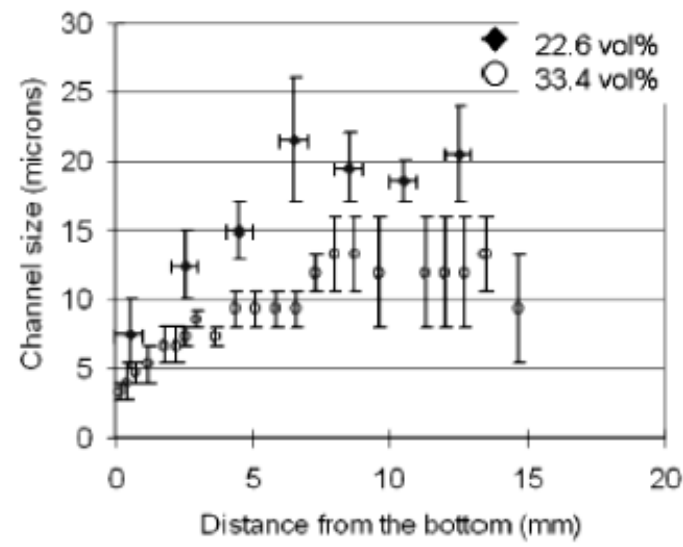

Fig. 1. Pore channel size versus distance from fracture surfaces of directionally solidified suspensions, showing results for two values of the particle concentrations in the suspension before freezing.

The cast bodies were sintered by heating at $5^{\circ} \mathrm{C} / \mathrm{min}$ to $1600^{\circ} \mathrm{C}$, and holding for $4 \mathrm{~h}$.

Cylindrical alumina samples were $30 \mathrm{~mm}$ in diameter and typically $15 \mathrm{~mm}$ in height using the camphene/alumina slurries containing $11.5,22.6,33.4,43.3$, and $52.3 \mathrm{vol} \%$ alumina. The alumina powder had a specific surface area of $8.6 \mathrm{~m}^{2} / \mathrm{g}$ and a median particle size of $400 \mathrm{~nm}$. The samples were directionally solidified by placing the mold on a heat sink and insulating the outside and top; thus, solidification initiated at the bottom of the sample and concluded at the top.

\section{Results and Discussion}

Fracture surfaces of sintered porous samples showed large channel pores in an otherwise dense matrix, which had been formed by the growth of camphene dendrites in the suspension. The channel size varied as a function of distance from the bottom of a directionally solidified sample, where the solidification initiated, as shown in Fig. 1, which is reproduced from Araki. ${ }^{9}$ The sample with $33.4 \mathrm{vol} \%$ alumina in the suspension had a smaller channel size than the sample with $22.6 \mathrm{vol} \%$ in the suspension. Solidification velocity was not directly measured, but Koch et $a l .{ }^{16}$ have shown that the frozen interface moves with $t^{1 / 2}$ for uniaxial freezing, implying that velocity $\mathrm{d} y / \mathrm{d} t$ varies with $1 / y$. Distance into the sample can be used as a surrogate for velocity. We use this to convert position to a "relative velocity." Thus, the data of Fig. 1 can be replotted as pore channel size versus relative velocity, in Fig. 2.

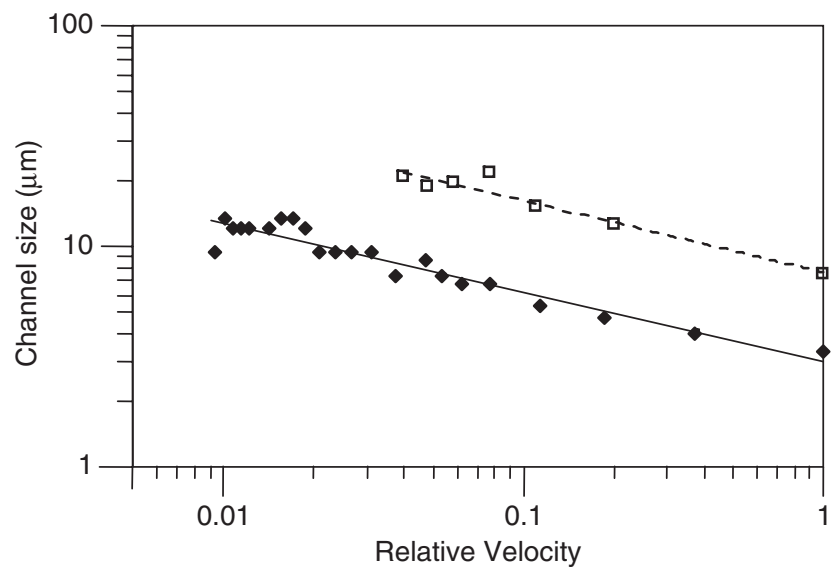

Fig. 2. Fracture surface channel size data replotted versus relative velocity of the solidification front. Open squares are for $22.5 \mathrm{vol} \%$ alumina; filled diamonds are for 33.4 volume $\%$ alumina in the suspensions before freezing. 


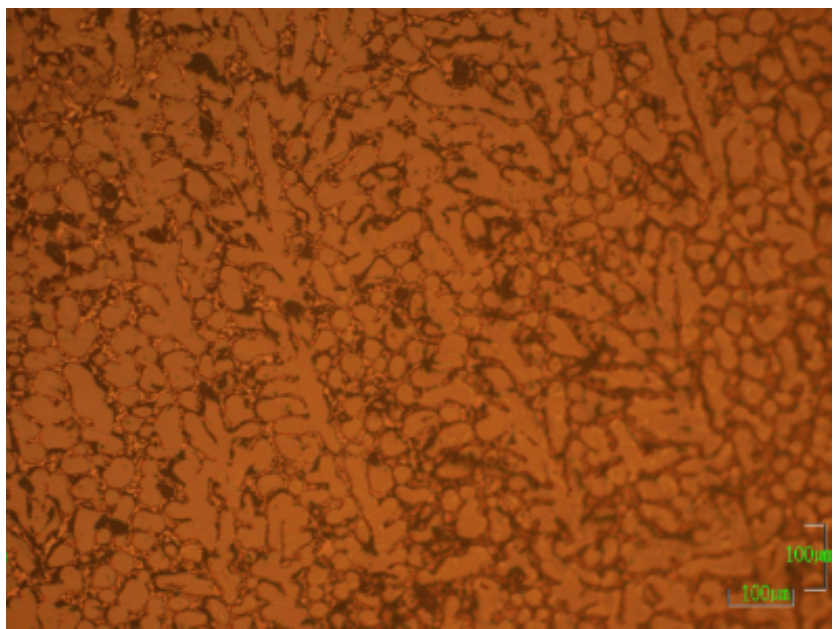

Fig. 3. Optical micrographs of a sample produced from $22.6 \mathrm{vol} \%$ $\mathrm{Al}_{2} \mathrm{O}_{3}$ suspension at a position $7 \mathrm{~mm}$ from the bottom of the directionally solidified casting. The dendritic features are channel pores filled by epoxy. Solidification direction is from the bottom to the top of the micrograph.

Here, we supplement the fracture surface observations with polished sections, in an effort to determine the dendritic channel pores more carefully. Sintered alumina samples made from suspensions originating with $22.6 \mathrm{vol} \%$ alumina, 33.4, 43.3, and $52.3 \mathrm{vol} \%$ were vacuum impregnated with Struers Epofix mounting epoxy to fill the pores and prevent breakage of the sample during polishing. The samples were then sectioned with a diamond blade and re-mounted in epoxy. They were polished using methods appropriate for alumina. Figure 3 shows a typical microstructure of the polished section of the porous alumina, where the dendritic features are the epoxy-filled channel pores. Measurements were made on the micrographs to determine the average SDAS for slices at six distances from the bottom of the sample (six values of " $y$ "), surveying the entire cross-section for two independent specimens. The $52.3 \mathrm{vol} \%$ alumina samples did not form dendrites and did not have channel pores. For the other specimens, the volume fraction of dendritic porosity was determined by image analysis.

The SDAS data appear in Fig. 4 as size versus position in the sample, measured from the location where solidification started. The arm spacing increases near the bottom of the sample, but begins to level off about halfway up the sample $(6-8 \mathrm{~mm})$. This is in qualitative agreement with the channel size data from frac-

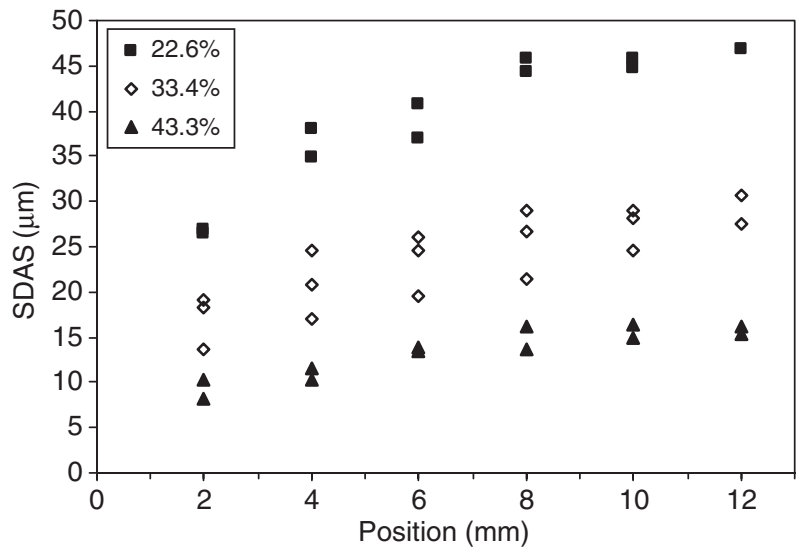

Fig. 4. Secondary dendrite arm spacing versus distance from the surface where freezing began, from measurements on polished sections of sintered specimens for suspensions with $22.6,33.4$, and $44.3 \mathrm{vol} \%$ alumina before freezing. Duplicate data is for independent specimens. Variations within a specimen are comparable with specimen-to-specimen variation.

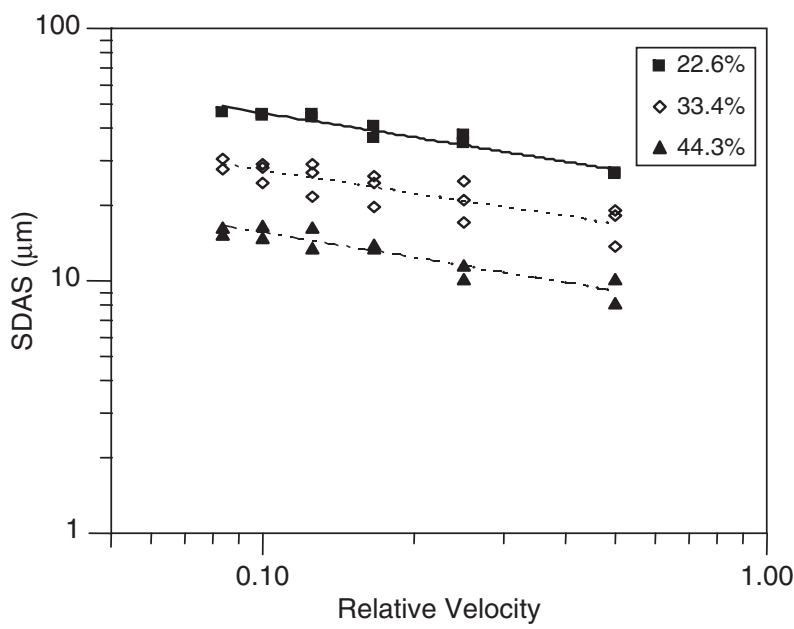

Fig.5. Secondary dendrite arm spacing versus relative velocity of the solidification front, for suspensions with $22.6,33.4$, and $44.3 \mathrm{vol} \%$ alumina before freezing.

ture surfaces in Fig. 1. Distances can be converted to a "relative velocity," defined as the inverse of distance, and are replotted in Fig. 5 for the suspensions with 22.6, 33.4, and $43.3 \mathrm{vol} \%$ alumina, respectively. In all cases, the data are well described by straight lines, as expected for Eq. (10), with the exponent $n \sim 0.33$. These data can be represented in the form of Eq. (10) by the following empirical fits to the data (here the units for the relative velocity are not expressed, as they are not directly measured):

$$
\begin{aligned}
& \lambda_{2}=22.1 v^{-1 / 3} \text { for the } 22.6 \mathrm{vol} \% \text { suspension } \\
& \lambda_{2}=13 v^{-1 / 3} \text { for the } 33.4 \mathrm{vol} \% \text { suspension } \\
& \lambda_{2}=7.2 v^{-1 / 3} \text { for the } 43.3 \mathrm{vol} \% \text { suspension }
\end{aligned}
$$

The nominal values for the constant $B$ in the fits above are plotted against volume fraction in Fig. 6. Notice that these three data appear to fall on a straight line, extrapolating to zero thickness around $\Phi=0.5$, which is close to the maximum particle concentration for jamming, $\Phi_{\mathrm{m}}$. It is intriguing that $B$ is proportional to $\left(\Phi-\Phi_{\mathrm{m}}\right)^{d}$ with $d=1$, although the data are too scanty to support this strongly. Dendrite pore sizes have been reported in the alumina-water system by Deville et al. $^{7}$ versus solidification rate and Koch et al. ${ }^{16}$ versus cooling rate. These appear to have a steeper dependence on solidification velocity, roughly $v^{-0.6}$, although the data set is sparse. Perhaps the camphene system differs from water, which forms lamellar dendrites.

The volume fraction of the alumina in the interdendritic regions was averaged from the five 22.6 and $33.4 \mathrm{vol} \%$ samples; in the $43.3 \mathrm{vol} \%$ samples, the interdendritic regions were nearly $100 \%$ dense due to sintering. To determine the critical volume

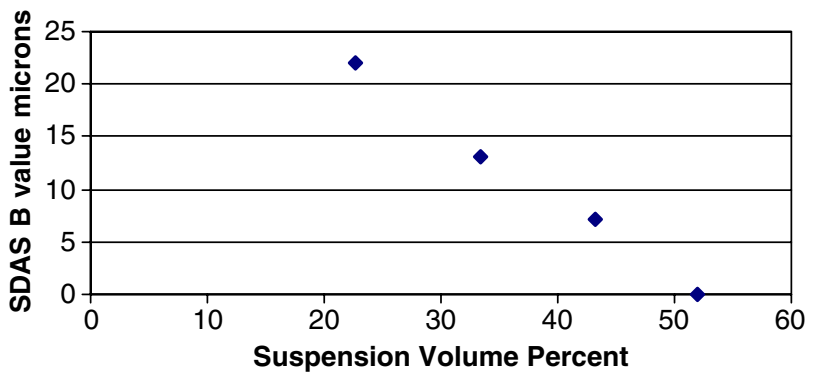

Fig. 6. Secondary dendrite arm spacing $B$ parameter versus volume percent ceramic powder in suspension. 


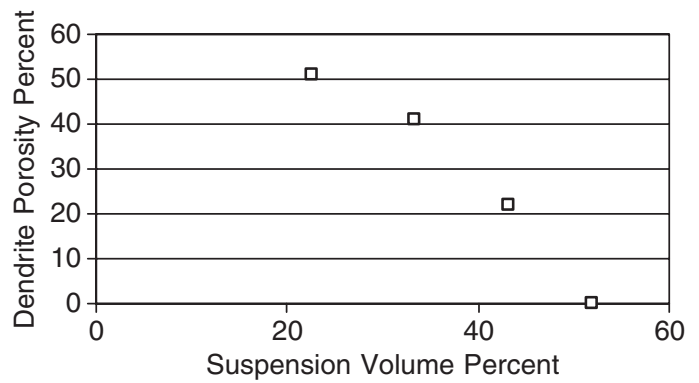

Fig. 7. Sintered density versus solid content in suspension before freezing.

fraction of alumina particles, $\Phi_{\mathrm{b}}$, we examine Fig. 7 and extrapolate the porosity from the dendrites versus solid content in slurries. By extrapolating these few data where $\Phi_{\mathrm{p}}$ was equal to $0, \Phi_{\mathrm{b}}$ is estimated to be about $0.55 \pm 0.01$.

We can relate this to $\Phi_{\mathrm{m}}$ using Eq. (8) by calculating the magnitude of osmotic and capillary term, $W$. For the case of camphene at $319 \mathrm{~K}$, the surface tension ${ }^{17}$ is $0.0044 \mathrm{~J} / \mathrm{m}^{2}$. The alumina powder used by Araki had a specific surface area of $8.6 \mathrm{~m}^{2} / \mathrm{g}$, so the equivalent particle radius is $88 \mathrm{~nm}$, so for this case $W=0.02$, or breakthrough occurs at a volume percent solids within $2 \%$ of $\Phi_{\mathrm{m}}$. From the approximate Eq. (7), we estimate $\Phi_{\mathrm{m}}=0.57 \pm 0.01$, while from Eq. (6) we obtain $\Phi_{\mathrm{m}}=$ $0.566 \pm 0.01$, or about the same value.

This value can be compared with rheological measurements of the upper Newtonian limit ${ }^{9}$ for the suspensions at $55^{\circ} \mathrm{C}$. These were fit by a Krieger-Daugherty equation with a maximum packing fraction $\Phi_{\mathrm{o}}$ of 0.61 , which expresses solidification by jamming for shear flow. The $\Phi_{\mathrm{m}}$ value inferred from the sintered samples, being the suspension concentration above which no dendrite pores are observed, underestimates the $\Phi_{\mathrm{o}}$ by about $4 \%$, although it is perhaps not surprising considering the quite different physical conditions of flow at the upper Newtonian limit and consolidation by a moving liquid-solid interface.

\section{Conclusions}

Solidification of the suspension medium for dilute suspensions can cause redistribution of the particles if the $\mathrm{S} / \mathrm{L}$ interface pushes the particles rather than engulfs them. This increases the particle concentration in the remaining liquid. The solid/liquid interface pushes particles until the osmotic pressure of the suspension exceeds the capillary pressure required to force the $\mathrm{S} / \mathrm{L}$ interface into the packed particles. The particle volume fraction at breakthrough is determined by the maximum packing of particles at the point of jamming, modified by a small term de- pendent upon particle size and surface tension. The predictions agree with observation for the alumina-camphene system within about $4 \%$.

Dendritic pores created in the suspension, preserved after sintering, display a secondary arm dendrite spacing that depends on apparent growth velocity according to a $1 / 3$ power law. The sizes of the dendrite pores are inversely proportional to particle volume fraction in the suspension.

\section{Acknowledgment}

We thank Prof. Jennifer Lewis of the University of Illinois for helpful suggestions concerning the osmotic pressure of suspensions.

\section{References}

${ }^{1}$ K. Araki and J. W. Halloran, "A New Freeze Casting Technique for Ceramics with Sublimable Vehicles,” J. Am. Ceram. Soc., 87 [10] 1859-63 (2004).

${ }^{2}$ B. E. Novich, C. A. Sundback, and R. W. Adams, "Quickset Injection Molding of High-Performance Ceramics"; pp. 157-64 in Ceramic Transactions, 26 Forming Science and Technology for Ceramics, Edited by M. J. Cima. American Ceramic Society, Westerville, $\mathrm{OH}, 1992$.

${ }^{3}$ M. A. Occhionero, B. E. Novich, and C. A. Sundback, "Forming of Complex High Performance Ceramic and Metallic Shapes"; U.S. Patent 5047181, 1991.

${ }^{4}$ S. W. Sofie and F. Dogan, "Freeze Casting of Aqueous Alumina Slurries with Glycerol,” J. Am. Ceram. Soc., 84 [7] 1459-64 (2001).

${ }^{5}$ T. Fukasawa, M. Ando, T. Ohji, and S. Kanazaki, "Synthesis of Porous Ceramics with Complex Pore Structure by Freeze-Dry Process," J. Am. Ceram. Soc., 84 [1] 230-2 (2001).

${ }^{6} \mathrm{~K}$. Araki and J. W. Halloran, "Porous Ceramic Bodies with Interconnected Pore Channels by a Novel Freeze Casting Technique," J. Am. Ceram. Soc., 88 [5] 1108-14 (2004).

${ }^{7}$ S. Deville, E. Saiz, R. K. Nalla, and A. P. Tomsia, "Freezing as a Path to Build Complex Composites," Science, 311, 515-8 (2006).

${ }^{8}$ D. R. Uhlmann, B. Chalmers, and K. A. Jackson, "Interaction between Particles and a Solid-Liquid Interface," J. Appl. Phys., 35, 2986-93 (1964).

${ }^{9} \mathrm{~K}$. Araki, "New Freeze Casting Technique for Ceramics, PhD Thesis, University of Michigan, 2004.

${ }^{10}$ J. J. Guo and J. A. Lewis, "Aggregation Effects on the Compressive Flow Properties and Drying Behavior of Colloidal Silica Suspensions," J. Am. Ceram. Soc., 82 [9] 2345-58 (1999).

${ }^{11}$ N. F. Carnahan and K. E. Starling, "Equation of State for Non-Attracting Rigid Spheres," J. Chem.Phys., 51 [2] 635-6 (1969).

${ }^{12}$ D. M. Smith, G. W. Scherer, and J. M. Anderson, "Shrinkage During Drying of Silica Gel," J. Non-Cryst. Solids, 188, 191-206 (1995).

${ }^{13}$ D. A. Porter and K. E. Easterling, Phase Transformations in Metals and Alloys. Section 3.3.5, 2nd edition, Chapman \& Hall, London, 1992.

${ }^{14}$ H. Biloni, "Chapter 9, Solidification"; pp. 500-79 in Physical Metallurgy, 3rd edition, Edited by R. W. Cahn, and P. Haasen. North-Holland Physics Publishing, Amsterdam, 1983.

${ }^{15}$ E. R. Rubenstein and M. E. Glicksman, "Dendritic Growth Kinetics and Structure II: Camphene," J. Crystal Growth, 112, 97-110 (1991).

${ }^{16}$ D. Koch, L. Andresen, T. Schmedders, and G. Grathwohl, "Evolution of Porosity by Freeze Casting and Sintering of Sol-Gel Derived Ceramics," J. Sol-Gel Sci. Technol., 26, 149-52 (2003).

${ }^{17}$ B. Bayender, N. Marasli, E. Chadirli, and M. Gunduz, "Solid-Liquid Interfacial Energy of Camphene," Mater. Sci. Eng., A270, 343-8 (1999). 